\title{
A Real-time Breast Hyperthermia Monitoring Scheme Based on Processing of Microwave Scattering Parameters with Deep Learning
}

This paper was downloaded from TechRxiv (https://www.techrxiv.org).

\section{LICENSE}

CC BY-NC-SA 4.0

SUBMISSION DATE / POSTED DATE

$30-12-2021 / 05-01-2022$

\section{CITATION}

Yilmaz, Tuba; Akinci, Mehmet Nuri; Girgin, Enes; Önal, Hulusi (2022): A Real-time Breast Hyperthermia Monitoring Scheme Based on Processing of Microwave Scattering Parameters with Deep Learning. TechRxiv. Preprint. https://doi.org/10.36227/techrxiv.17707106.v1

$\mathrm{DOI}$ 


\title{
A Real-time Breast Hyperthermia Monitoring Scheme Based on Processing of Microwave Scattering Parameters with Deep Learning
}

\author{
Enes Girgin, Hulusi Önal, Tuba Yilmaz,Member IEEE and Mehmet Nuri Akıncı
}

\begin{abstract}
This paper presents a real-time and non-invasive method based on scattered electric fields to ensure the proper heating in breast medium during microwave hyperthermia (MH) treatment. The proposed method comprises application of deep neural network (NN) to scattered field data for non-invasive detection of thermal anomalies in the target medium. Microwave dielectric properties (DPs) of biological tissues are known to change with temperature. It is also well known that the electromagnetic wave propagation is governed by the DPs of a medium. The proposed deep learning (DL) method uses scattered electric field data to exploit the temperature-dependent DP change in the medium for thermal monitoring. The sensitivity of the model is analyzed by re-performing training and testing using modified data with various added noise levels. Training is evaluated using Jaccard score metrics. A neighborhood algorithm is introduced to further improve the accuracy of the model. Once the model is finalized, validation is performed with unused data. The proposed model is able to perform predictions with $92.9 \%$ accuracy.
\end{abstract}

Index Terms-Microwave breast hyperthermia, microwave breast imaging, deep learning, non-invasive temperature monitoring

\section{INTRODUCTION}

$\mathbf{M}$ ICROWAVE HYPERTHERMIA (MH) is a non-invasive breast cancer treatment technique used either solo or in combination with radiotherapy and chemotherapy, also referred as mild hyperthermia [1], [2]. The goal of sole MH treatment is to increase the target tissue temperature above $42^{\circ} \mathrm{C}$ to enable cell necrosis in the malignant tumor region [3]. While doing so, the healthy breast tissue surrounding the malignant tumor should remain at a safe temperature. As a conjunction therapy the MH is used to increase the effectiveness of other therapies by heating the target tissue region to a desired temperature, typically few degrees higher than normal body temperature, while keeping the remaining tissue at a safe temperature [4], [5]. Ensuring the proper heating at the target tissue and preventing hot-spots in the healthy tissue with a non-invasive approach remains a challenge.

To address this need, many different studies have been reported in the literature for monitoring of the temperature distribution during microwave hyperthermia treatment. One

Manuscript received xxx; revised xxx. This work was supported by the Scientific and Technological Research Council of Turkey under grant agreement $118 \mathrm{~S} 074$.

The authors are with the Department of Electronics and Communication Engineering, Istanbul Technical University, Istanbul, 34469 Turkey (e-mail: akincime@itu.edu.tr.)

Color versions of one or more of the figures in this paper are available online at http://ieeexplore.ieee.org. such method is microwave radiometry, proposed in early 1970 s [6]. Radiometry aims to detect the temperature-dependent radiations including but not limited to black-body radiation for breast cancer diagnosis and non-invasive temperature control during hyperthermia treatment [7], [8]. However, temperature monitoring with microwave radiometry has several challenges. It is known that low level of electromagnetic (EM) thermal noise is a disadvantage for temperature detection. Due to the system noise and EM interference, detection of thermal noise with radiometry is cumbersome [9]. To enable thermal detection with a radiometry system, there is a need to optimize and stabilize the entire system.

Another method proposed for non-invasive temperature monitoring is microwave radar based imaging. Similar to $\mathrm{MH}$, the microwave imaging (MI) based temperature monitoring methods exploits the dielectric property (DP) discrepancy. This discrepancy forms during $\mathrm{MH}$ treatment due to temperature variations. Although the DPs does not significantly change with temperature, the variation is sufficient for temperature detection with MI [10]. For radar based MI, DP variation effects the reflections which can then be detected by receivers [11]. In [12], radar based non-invasive temperature monitoring along with phantom experiments is reported.

Yet another microwave based temperature monitoring method is presented in [13] where a wearable microwave thermometer for non-invasive and real-time body temperature monitoring is proposed. An instantaneous temperature estimation was performed with a probe placed on the skin [14].

Among other reported studies, magnetic resonance (MR) guided microwave hyperthermia is shown to be a clinically feasible method [15]. In [16] MR is used clinically for temperature monitoring during hyperthermia treatment. However, this system is costly and difficult to implement. Therefore, new methods are being investigated.

It is well known that MI exploits inherent dielectric property discrepancies [17], [18]; therefore, it can also detect the temperature-dependent change in DPs of biological tissues. In literature, using MI during hyperthermia treatment was experimentally demonstrated in [19], [20]. Although several studies, including but not limited to MI, radar based MI, radiometry, are reported for temperature monitoring during $\mathrm{MH}$, there is still a need to provide a reliable method that can monitor the temperature in the heterogeneous biological tissue. The proposed methods fails to consider the complex nature of the biological tissue and limited with few heating scenarios. Thus, the newly proposed method should be application spe- 


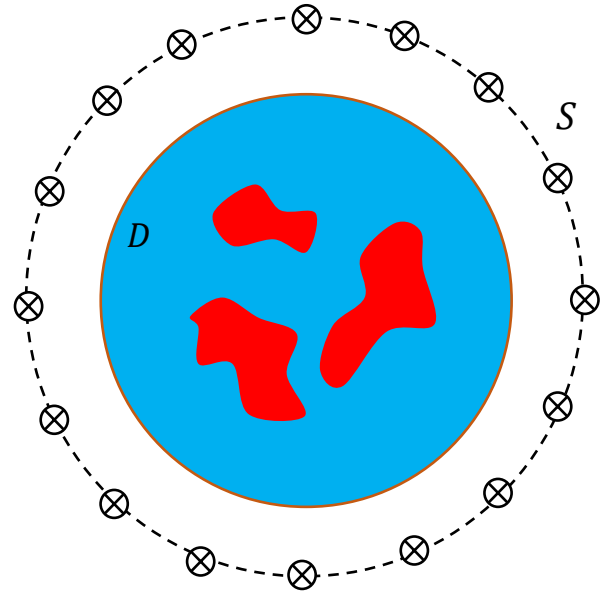

Fig. 1: General geometry for 2D electromagnetic (EM) scattering problem.

cific and it should be statistically validated under different heating scenarios. In this paper, we are proposing a deep learning (DL) approach for real-time and non-invasive thermal monitoring during the $\mathrm{MH}$ treatment. The DL model designed to process the microwave fields scattering from a realistic breast phantom modified to include several tumor inclusions with thermal anomalies as well as hot spots in surrounding healthy tissues. Instead of a thermal map retrieval, the proposed method focuses on detection of an thermal anomaly. Being a DL based evaluation method, the proposed technique is statistically validated for many different scenarios. To the best of authors' knowledge, this study is the first to (I) apply DL to microwave scattering data for thermal monitoring, (II) change the non-invasive approach from temperature mapping to thermal monitoring.

The remainder of this paper is organized as follows: Section 2 and 3 describe data generation and data pre-processing steps, respectively. Utilized method is given in section 4 . Sections 5 describes the structure of the deep learning model, the training and testing results, and the validation of results. Finally, conclusions are drawn in section 6.

\section{DATA ACQUISITION}

\section{A. Scattering Simulations}

Direct scattering solvers can be used to simulate measurement data in the scattering problems. Using numerical solution methods is essential for such simulations since there is no closed form solution for arbitrary shaped or inhomogeneous objects. Thus, the Electric Field Integral Equation (EFIE) describing the EM wave scattering behavior must be solved with the numerical techniques. One such technique is Method of Moments (MoM), which is based on discretization of the computation domain by means of pulse basis functions. Note that computation domain includes the buried object and the background medium.

General configuration of 2D EM scattering problem is shown in the Fig. 1. In this figure, $D$ indicates the computation domain, $S$ denotes the region with transmitter and receiver antennas. Based on this figure, the domain equation used for calculation of total field, can be written as follows [21],

$$
E\left(r, r^{\prime}\right)=E_{i}\left(r, r^{\prime}\right)+k_{0}^{2} \int_{D} G\left(r^{\prime \prime}, r\right) \xi\left(r^{\prime \prime}\right) E\left(r^{\prime \prime}, r^{\prime}\right) d r^{\prime \prime}
$$

where $r, r^{\prime \prime}$, and $r^{\prime}$ are position vectors which belong to source cells in domain $D$, observation cells in domain $D$, and transmitter antennas in domain $S$, respectively. $E$ denotes the total electric field and $E_{i}$ denotes the incident electric field. Note that antennas producing $E_{i}$ are modelled with line sources in this work. $G\left(r^{\prime \prime}, r\right)=\frac{i}{4} H_{0}^{(1)}\left(k_{b}\left|r-r^{\prime \prime}\right|\right)$ denotes the Green's function of 2D EM problem, and $k_{b}$ is the wave number in the background medium. Lastly, $\xi$ is contrast vector, which represents the difference in complex dielectric constants between point $r$ and the background medium.

$$
\xi(r)=\epsilon_{r}(r)-\epsilon_{r b}
$$

The integral equation in (1) is discretized by dividing domain $D$ to $N$ cells, then a $N$-by- $N$ matrix equation is obtained. The total field can be computed via the solution of this equation system. Then, the scattered field can be easily determined by the following scattering equation [22].

$$
E_{s}\left(r^{\prime \prime}, r^{\prime}\right)=k_{0}^{2} \int_{D} G\left(r^{\prime \prime}, r\right) \xi(r) E\left(r, r^{\prime}\right) d r, r^{\prime \prime} \in S
$$

The scattered field matrix is a $R$-by- $T$ matrix, where $R$ denotes number of receivers, and $T$ denotes number of transmitters. In a practical scenario, this matrix is obtained by sequential measurements carried out by assigning an antenna as transmitter and the remaining ones as receiver at time $t$. In this work, a configuration with 64 line sources, positioned equidistantly on a circle having radius of $7.5 \mathrm{~cm}$, were configured for data generation. Therefore, a scattering matrix $E_{s}$ with $64 \times 64$ elements as complex numbers were obtained.

\section{B. Modelling of the computational domain}

Realistic breast models described in [23] was obtained from the Cross-Disciplinary Electromagnetics Laboratory in the University of Wisconsin-Madison. A heterogeneously dense model with 062204 ID number was selected, and 2D cross section was obtained from $96^{\text {th }}$ vertical slice of the 3D model. The resolution of the model was $2 \mathrm{~mm}\left(\sim \frac{\lambda}{10}\right)$ which can be considered as a sufficient value for the numerical simulations. Dielectric constant $\left(\varepsilon_{r}\right)$ and conductivity $(\sigma)$ distributions are given in Fig. 2a and 2b, respectively.

During simulations, relative dielectric constant and conductivity value of the matching medium were $\epsilon_{r}=2.6$ and $\sigma=0(\mathrm{~S} / \mathrm{m})$, respectively [24]. Single frequency analysis were performed, and the operating frequency was $2 \mathrm{GHz}$.

DP distributions given in Fig. 2 are considered as reference values corresponds to DPs for temperature level of $37^{\circ} \mathrm{C}$, which is referred as the cool state. Since hyperthermia is a cancer treatment, considered scenario must include a tumor as seen in Fig. 2a-2b. Here, the tumor is fixed to the position of $(2,0) \mathrm{cm}$. It is assumed that the DP of the patient's breast is obtained previously through an MRI scan. Thus, the breast 


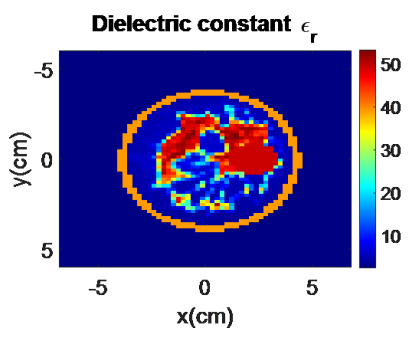

(a)

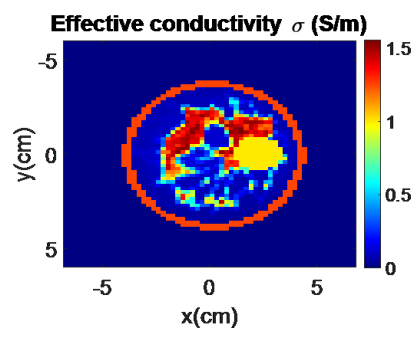

(b)
Fig. 2: Dielectric property distribution in the realistic breast cross-section at $2 \mathrm{GHz}$. (a) Relative dielectric constant $\left(\varepsilon_{r}\right)$, (b) effective conductivity $(\sigma)$.

model given in Fig. 2a-2b is assumed to be known before treatment. The goal is to design a patient-specific neural network (NN) before the application of the hyperthermia procedure to use it during hyperthermia treatment for thermal monitoring. During application of hypertermia, the designed patient-specific $\mathrm{NN}$ has to detect the anomaly within the breast medium and warn the user. The anomalies can be defined as (1) presence of hot spots at undesired locations within the healthy breast tissues, or (2) inability to reach the designated temperature at the breast tumor. If anomaly (1) occurs due to the failure of proper hyperthermia application, the hot spots can potentially be at any location within the breast medium. Therefore, the designed NN should "learn" all possibilities. Thus, in this work we considered up to three randomly located hot spots in the breast. Here, hot spots were assumed to be circular with a randomly assigned radius value among [0.8 0.9 $\left.\begin{array}{lll}1 & 1.1 & 1.2\end{array}\right] \mathrm{cm}$. Once the models were formed, sample-specific scattering problem was solved.

The thermal anomalies and the normal temperatures were modelled with the temperature-depended DPs. However, temperature-dependent DPs of a small number of animal tissues were tabulated in the literature at microwave frequencies in-vitro such as liver, fat, and blood [10], [25]. Also, DP of in-vitro breast tumor at microwave frequencies were reported in literature [26], [23]. Due to the unavailability of the temperature-dependent DPs of breast tissues, other reported temperature-dependent tissue DPs can be employed. This can be justified since the biological tissues can be characterized as low, intermediate, high water content tissues. It is expected that the tissues at the same category shows similar DP behaviour. Based on this assumption, since breast tumor, fibroglandular tissue, and liver tissues all belong to high water content tissues, temperature dependency of said breast tissues was assumed to be similar to liver tissue. Both studies [10], [25] show that temperature-dependent DPs of liver at $2 \mathrm{GHz}$ frequency are almost linearly dependent to temperature from 37 to $45^{\circ} \mathrm{C}$. This temperature range is considered as a suitable interval for hyperthermia applications [27]. At $2 \mathrm{GHz}$, relative dielectric constant, conductivity of the liver is reported as $\epsilon_{r}=49.41$, $\sigma=1$ and $\epsilon_{r}=48.48, \sigma=1.1$ at $37^{\circ} \mathrm{C}$ and $45^{\circ} \mathrm{C}$ for lower and upper bound of the temperature levels, respectively [10]. Therefore, it can be stated that there is a decrease in dielectric constant by $\% 2$ and increase on conductivity by $\% 10$ for liver tissue, and, thus, for the high water content tissues. In accordance with these assumptions, the temperature dependent dielectric constant and conductivity of the breast tissues is given as,

$$
\begin{array}{r}
\epsilon_{r}(r, T)=\epsilon_{r}\left(r, 37^{\circ}\right) \times\left(1-\frac{T-37^{\circ}}{8^{\circ}} \times \frac{2}{100}\right) \\
\sigma(r, T)=\sigma\left(r, 37^{\circ}\right) \times\left(1+\frac{T-37^{\circ}}{8^{\circ}} \times \frac{10}{100}\right)
\end{array}
$$

As stated before, the target in hyperthermia therapy is to heat only the tumor tissue and heating of the surrounding healthy tissues is not desired. Thus, a threshold temperature value must be determined for each tissue. In this work, $38.5^{\circ} \mathrm{C}$ was selected as the maximum allowed temperature for healthy tissues, and $42^{\circ} \mathrm{C}$ was selected as the minimum allowed temperature for tumor tissue. Therefore, goal of this work is ensuring the proper application of hyperthermia through detection of the temperature anomalies in the breast tissue, where the anomaly is defined as temperatures higher than $38.5^{\circ} \mathrm{C}$ for healthy tissues and lower than $42^{\circ} \mathrm{C}$ in the tumor region. When an anomaly occurs, the temperature of a "hot" location may vary. Here, after selecting random locations for each "hot" location the temperature is randomly assigned between $37^{\circ} \mathrm{C}-45^{\circ} \mathrm{C}$. Then, the electrical properties in "hot" case is found with (4) and a MoM solution is performed for "hot" case. Here, it is important to emphasize the two cases to convey which conditions were considered as anomalies.(1) if the temperature of a random hot location at healthy tissue is lower than $38.5^{\circ} \mathrm{C}$, it was assigned as normal or a random hot location. Similarly, if the temperature of the tumor tissue was more than $42^{\circ} \mathrm{C}$, it is assigned as normal. (2) If the temperature of healthy region was more than $38.5^{\circ} \mathrm{C}$ or the temperature of the tumor is less than $42^{\circ} \mathrm{C}$, it is assigned as a thermal anomaly. Lastly, Fig. 3 gives a step by step presentation of the proposed hypertermia monitoring in order to summarize the said details.

\section{DAta PREPRocessing}

In this section, the data preprocessing steps required for the convolutional neural network $(\mathrm{CNN})$ model to work are explained.

The input of the CNN is the difference between the "hot" and the "cold" states. For each state the bi-static scattering matrix is composed of complex numbers and has a size of $64 \times 64$. The CNN works only with real inputs; thus, the obtained matrix is fist separated to its real and imaginary parts and then these two parts are combined into one matrix with $64 \times 128$ size. This input includes both domain $\mathrm{S}$ and D, shown in Fig.1. Since the goal is to monitor the breast medium, only computational domain $\mathrm{D}$ can be considered by removing the matching medium (S domain) from the whole domain. Remaining image contains 1319 pixels. Since the approach is to design a different CNN for each pixel, the number of the pixels has to be reduced. For this purpose, each cell in the original breast image (with $68 \times 60$ size corresponding to 1319 breast pixels) is labeled as "0" or " 1 ". The relationship between the temperature and DPs of the 


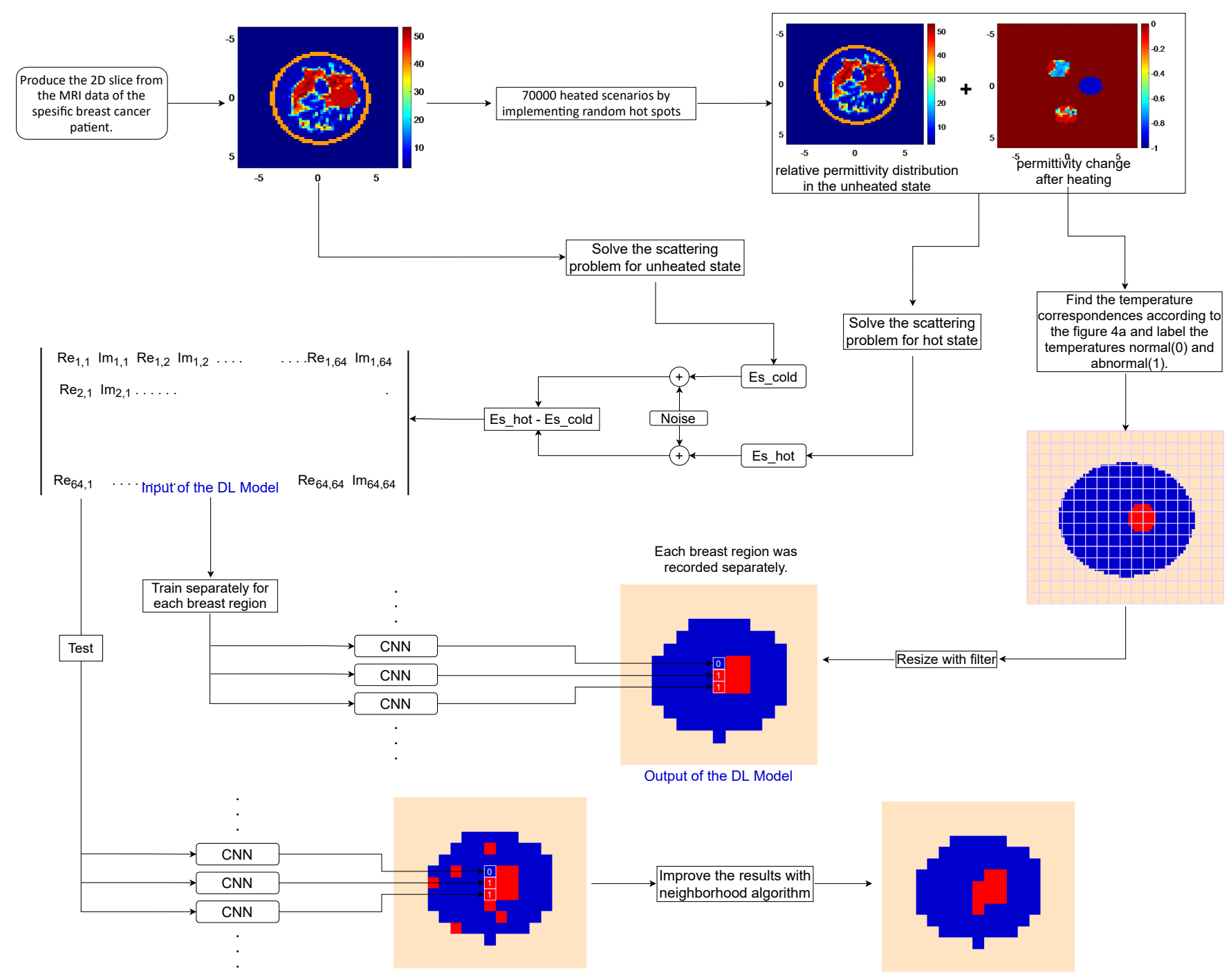

Fig. 3: Flowchart of the thermal monitoring algorithm.

liver are presented in Fig. 4. As shown in Fig. 4a the cell temperature increases by $4{ }^{\circ} \mathrm{C}$ in response to a $1 \%$ decrease in relative permittivity of liver tissue. Based on the percent change in this figure, the corresponding temperature for each pixel was calculated. The label "0" was used to indicate a normal condition corresponding to $T<38.5^{\circ}$ for healthy or benign tissues and/or $T>42^{\circ}$ for malignant tumor tissues. "1" was used to indicate an anomaly corresponding to $T>38.5^{\circ}$ for healthy or benign tissues and/or $T<42^{\circ}$ for malignant tumor tissues.

Next, a $4 \times 4$ filter with a slide of $4 \times 4$ is used to reduce the number of cells, hence the number of CNNs. Obtained image contained $17 \times 15$ pixels. In other words, each $4 \times 4$ image is mapped to a single pixel in the output. The pixels of the resulting $17 \times 15$ image is re-labeled with "0" and "1". The value of each pixel in $17 \times 15$ image is assigned such that it becomes "1", if there is at least one many "1" label in the corresponding $4 \times 4$ pixel of original image; otherwise, it was assigned as "0" (i.e. all labels are "0" in the corresponding $4 \times 4$ pixel of original image). Note that, a $\mathrm{D}$ domain with rather coarse boundary was obtained after the application of the filter. This is due to the adopted assignment strategy where a cell in the output image $(17 \times 15$ image has to be counted as a breast cell if there is at least one cell of breast (D domain) in its corresponding $4 \times 4$ image. Since the assignment of hotspots were random and each cell in $68 \times 60$ image may be labeled with "1"; thus, any cell at $17 \times 15$ image may be labeled as "1". For those cells in $17 \times 15$ (S domain), which are mapping only from the background, they become the background of $17 \times 15$ image since those are always " 0 ".

Finally, After applying the filter, the number of breast cells becomes 84 in the $17 \times 15$ image. To illustrate the process, an example of an original labeled breast image $(68 \times 60)$ and the image obtained after filtering $(17 \times 15)$ are shown in Fig. 5 .

\section{Methodology}

Balanced data is desired for training of an $\mathrm{NN}$ to prevent bias. Forming a single $\mathrm{CNN}$ with the $84 \times 1$ vector output is therefore not desirable since the training data will be unbalanced with too many "0" labels and too few "1" labels. It 


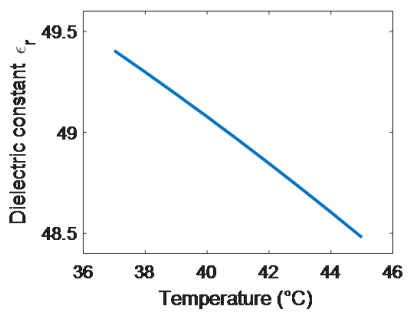

(a)

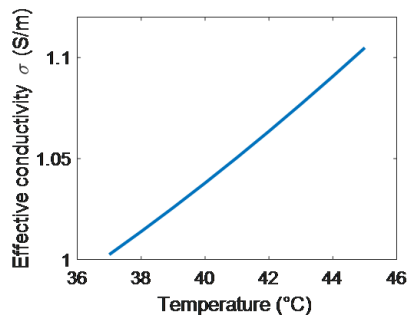

(b)
Fig. 4: Temperature-dependent dielectric properties (DPs) of liver tissue at $2 \mathrm{GHz}$. (a) Relative dielectric constant, (b) effective conductivity.

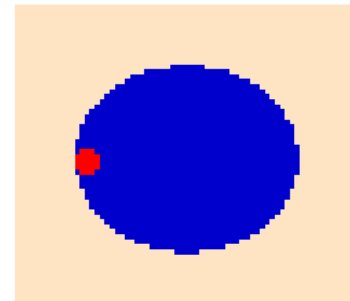

(a) Size: $68 \times 60$ pixels

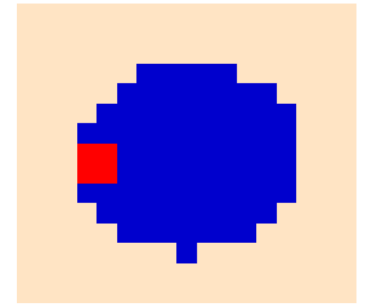

(b) Size: $17 \times 15$ pixels
Fig. 5: Reducing the number of pixels of the breast image (a) First Image (b) Image after filtering

should be noted that in general number of anomaly labels are quite lower than number of normal labels. To overcome this problem, a separate training was performed for each of the 84 cells. The CNN model structure described in the next section was kept constant, but different weights were determined for each cell. This approach enabled the training of 84 cells separately and $84 \mathrm{CNNs}$ different weights were obtained. The input of each model remained unchanged which was $64 x 128$ scattered electric field matrix, and a single binary output. With this approach 84 different training were conducted for 84 CNNs.

Assuming that the a diagnosed patient would have the MRI based DP distributions given in Fig. 2, a total number of 50000 "fictual breast cases" was obtained through random placements of heat anomalies to the DP distributions. However, as stated earlier, the number of " 0 " outputs for each region was greater than those with output of "1", which denotes the anomaly. To form a balanced dataset, the total number of outputs labeled with "1" was determined and same number of outputs with "0" label was randomly chosen for each cell. Through this approach, a balanced dataset for each region was obtained. As a result of this process, each of the 84 cells had a different number of datasets. The number of training data with "0" and "1" labels were equal for each cell that was used for training cell-specific model.

Using the CNN structure described in the next section, each of 84 cells in breast was trained and the results were recorded. Next, Gaussian noise was added to the model input (which is the difference between the "hot" and "cool" states) and testing were performed for $60 \mathrm{~dB}, 70 \mathrm{~dB}$ and $80 \mathrm{~dB}$ signalto-noise ratio (SNR) values. After completion of the training processes, 20000 breast scenarios, which were never seen by the $\mathrm{CNN}$ in the training phase, were used for validation. One can consider the first 50000 scenario can be considered as the models derived from real MRI used for training of CNNs. On the other hand, 20000 breast scenarios can be considered as the scenarios during the application of hyperthermia treatment to the patient.

Two different evaluation metrics are employed for assessing the quality of the predicted cells/images. While training and test of 50000 breast scenarios (for which the training are performed on), "accuracy of prediction for each of 84 cells" is used as evaluation metric. During the evaluation of the 20000 breast scenarios (unseen to the $\mathrm{CNN}$ in training phase), the predicted image formed first instead of assessing the prediction of single pixels and "Jaccard Index for each image" is used as evaluation metric.

\section{MODEL AND RESULTS}

CNN structure, shown in Fig. 6, was used in the model. The model consisted of convolution, batch normalization, and max pooling layers. ReLu activation function was used in these layers. After applying 5 layers, flatten was applied and a fully connected layer was added. The output of the model is a single binary number namely "0" or "1". Sigmoid function was used as the activation function in the last layer.

Training of all 84 CNNs lasted approximately 22 hours. The training was performed in Python language with using Tensorflow 2 libraries. The number of data for each pixel was randomly divided into $80 \%$ and $20 \%$ for train and test, respectively. The average train accuracy of over $84 \mathrm{CNNs}$ was 0.988. Binary cross entropy was used as the loss function. Average train loss was 0.03 . Average test accuracy was 0.89 and average test loss was 1.62. The average training data used out of 50000 data for each cell was 13398 and the average test data was 3350. Note that, as mentioned earlier, the amount of data may vary from pixel to pixel. In general the number of "1"s are quite less than "0"s. To equalize them, the some of "0"s are randomly discarded. Moreover, the hot spots are randomly distributed and thus the number of "1"s are different from pixel to pixel. Thus, for each $84 \mathrm{CNN}$, the number of training and test data varies. The accuracy graph of the training and test results is shown in Fig. 7.

After, the noise-free dataset was processed, trainings were also conducted with noisy datasets. In these trainings, the output of the model remained unchanged, and different levels of Gaussian noise was added to the input values. The same level of noise was added to the scattered electric field matrix in the "hot" state and in the cool state. Then, the difference of these two matrices was taken as in the noise-free training. Noisy results can be seen in Table. I

The parameters of the 84 trained models were recorded in 84 different model files. For validation, a new 20000 scenario was generated and data pre-processing steps were applied. The purpose was to use a new dataset that has never been used in the training and test sets for validation purposes. The performance of this set was evaluated using the Jaccard index. The 20000 scattered electric field data was given as input to 


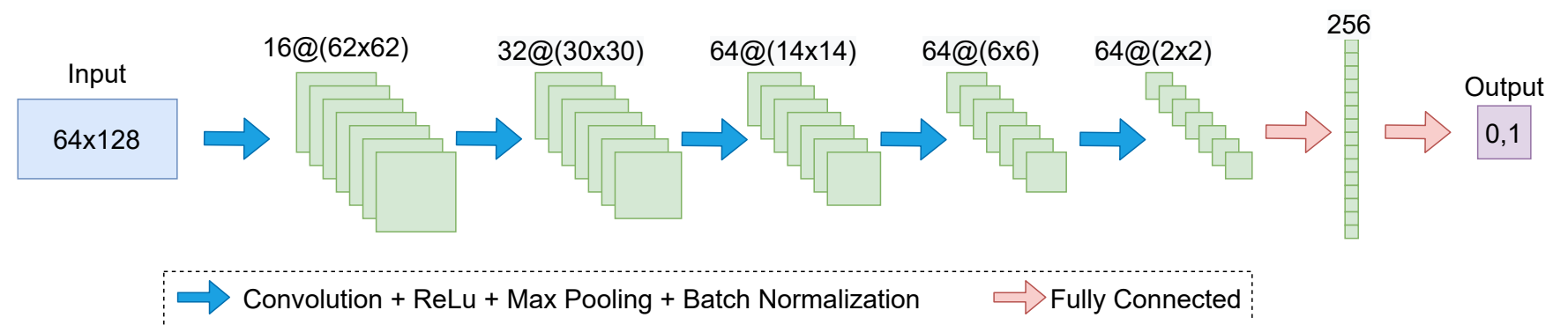

Fig. 6: Broad structure of the convolutional neural network (CNN) designed for thermal anomaly classification.

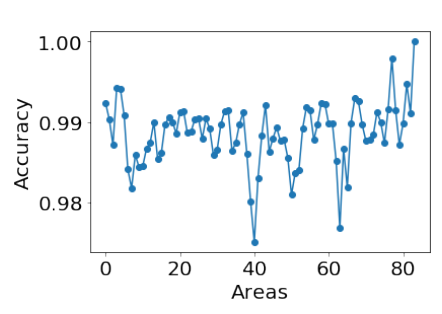

(a)

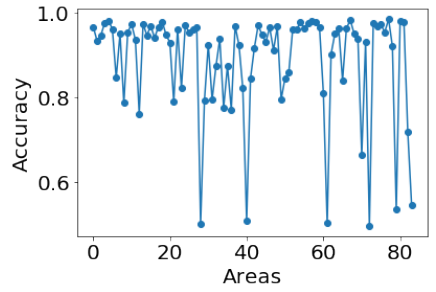

(b)

Fig. 7: Accuracy scores after training and test (a) Train accuracy (b) Test accuracy.

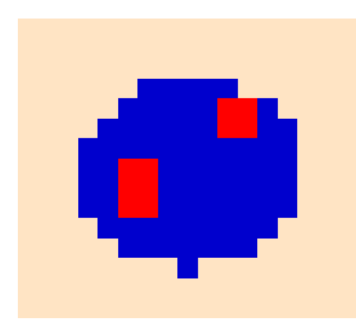

(a) Labeled Image

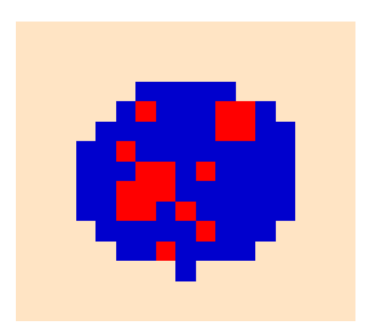

(b) Predicted Image

Fig. 8: An example of labeled and predicted images

84 different model files. The models made their predictions and created a vector with 84 elements. As a result, 20000 84 -element vectors were obtained. Next, the same files were labeled according to their permittivity values. The labeled image was the original image. The differences between the predicted model image and the labeled image were examined. An example can be seen in Fig. 8.

When 20000 validation data were examined, the similarity between the images for each was measured with the Jaccard index. The average Jaccard index was found to be 0.835 . The Jaccard index histogram can be seen in the Fig. 11a. The generated 20000 data were also used for noisy train models. The same operations were performed with the noisefree condition. Jaccard Score histograms can be seen in section V-A, Fig. 11.

\section{A. Enhancement of Results}

As it can be seen in Fig. 8, some undesirable spots emerge in predicted images. We expect that the heating anomalies should be clustered during hyperthermia. When the predictions

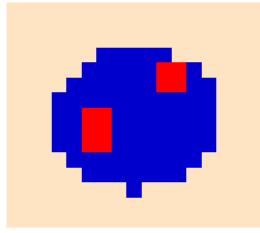

(a) Labeled Image

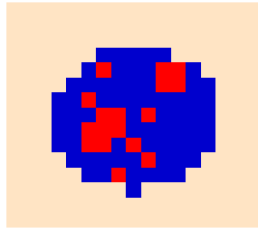

(b) Predicted Image

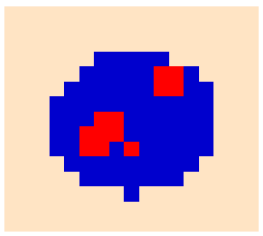

(c) Last Image
Fig. 9: An example of labeled, predicted and last images

compared to the labeled images, the scattered anomalies in predicted images was mispredicted, confirming our assumption. Eliminating these points would increase accuracy and the Jaccard score. Therefore, the neighborhood of the red regions predicted as 1 was examined. If no neighbor of the region predicted as 1 was predicted 1 , then that point was considered incorrect and replaced with 0 . If only one neighbor of the region is 1 , this region is again replaced with 0 . That is, if at least two other neighbors are 1 , the prediction is accepted as correct. This process was applied to 20000 data. As a result, accuracy increased from 0.89 to 0.929 . Jaccard score also increased from 0.835 to 0.87 . An example of the images formed as a result of this process is shown in Fig. 9. Fig. 11c presents the Jaccard histogram after processing.

The same operations were repeated for the noisy situation. The mean values of the noisy situation can be seen in Table. I, and the Jaccard score histograms before and after this process can be seen in Fig. 11.

\section{CONCLUSION}

In this paper, we proposed a deep learning-based noninvasive method to display in $2 \mathrm{D}$ whether the tissue is at the desired temperature values in the $\mathrm{MH}$ treatment. Many methods have previously been proposed for temperature monitoring during the $\mathrm{MH}$ treatment. We aimed to determine the temperature easily and effectively with the proposed method, which can only be applied using the scattered electric field data in the cool state and the heated state. In the first stage, the data was generated for deep learning. Then training and testing procedures were performed to design the network. Next, the results were obtained. Noise was added to the generated input data and the method was validated by applying the same procedures for noisy situations. The success of the method was measured using the Jaccard metrics. 
TABLE I: All Results

\begin{tabular}{l|l|l|l|l} 
SNR & Mean Train Accuracy & Mean Test Accuracy & Jaccard Score of Predicted Data & Jaccard Score after Last Process \\
\hline $60 \mathrm{db}$ & 0.982 & 0.834 & 0.589 & 0.695 \\
$70 \mathrm{db}$ & 0.985 & 0.857 & 0.738 & 0.816 \\
$80 \mathrm{db}$ & 0.986 & 0.861 & 0.821 & 0.856 \\
Noise Free & 0.988 & 0.89 & 0.835 & 0.870
\end{tabular}

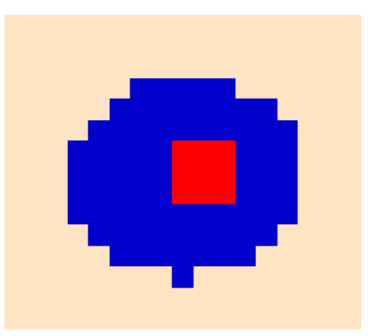

Fig. 10: A sample of labeled image

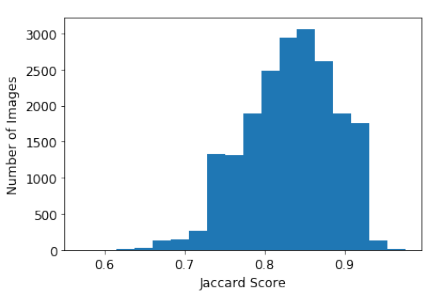

(a)

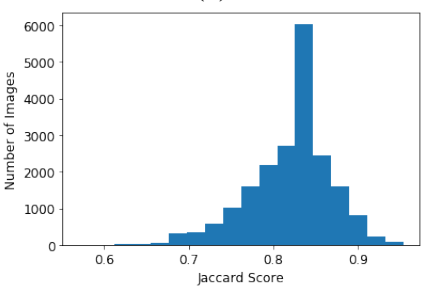

(e)

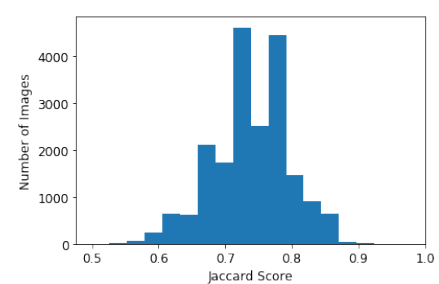

(i)

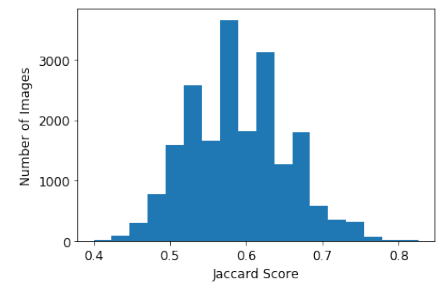

(m)

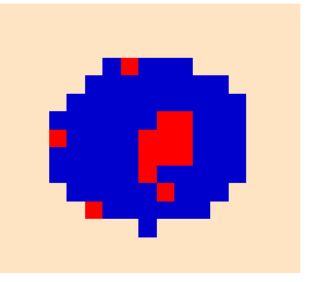

(b)

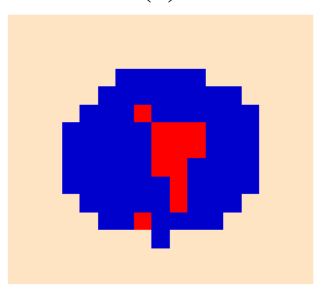

(f)

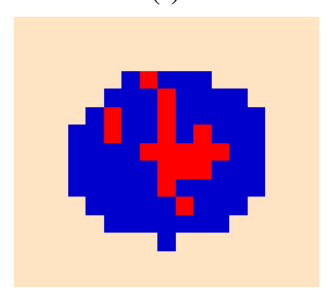

(j)

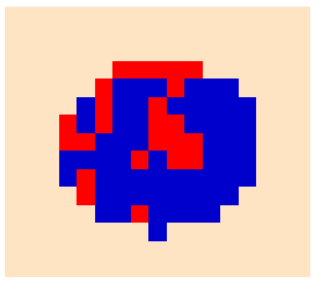

(n)

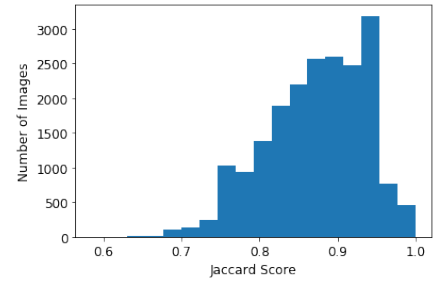

(c)

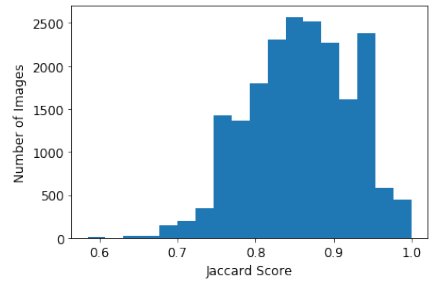

(g)

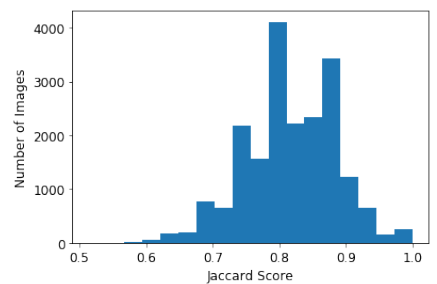

(k)

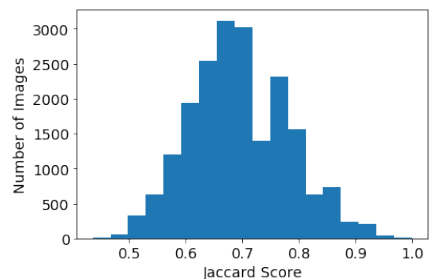

(o)

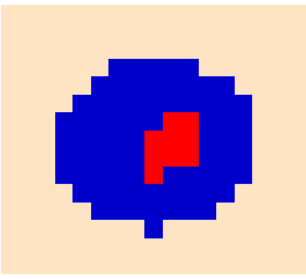

(d)

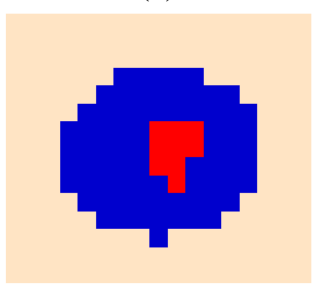

(h)

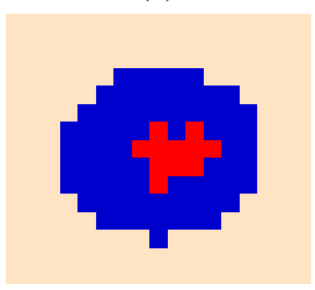

(1)

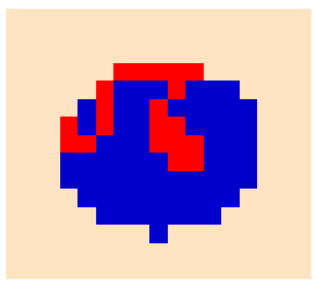

(p)

Fig. 11: The predicted images of the sample image in Fig. 10 by the noisy and noiseless training models and Jaccard histograms of the last test set. (a)-(d) Noise-Free model, (e)-(h) Noisy model with 80dB SNR, (i)-(l) Noisy model with 70dB SNR, (m)-(p) Noisy model with 60dB SNR. First two columns show the Jaccard histograms of final test set and predicted image. Last two columns show Jaccard histograms of final test set after neighborhood algorithm and predicted image.

\section{REFERENCES}

[1] M. Converse, E. J. Bond, B. Veen, and C. Hagness, "A computational study of ultra-wideband versus narrowband microwave hyperthermia for breast cancer treatment," IEEE transactions on microwave theory and techniques, vol. 54, no. 5, pp. 2169-2180, 2006. 
[2] J. E. Robinson, M. J. Wizenberg, and W. A. McCready, "Combined hyperthermia and radiation suggest an alternative to heavy particle therapy for reduced oxygen enhancement ratios," Nature, vol. 251, no. 5475, pp. 521-522, 1974

[3] P. T. Nguyen, A. Abbosh, and S. Crozier, "Microwave hyperthermia for breast cancer treatment using electromagnetic and thermal focusing tested on realistic breast models and antenna arrays," IEEE Transactions on antennas and propagation, vol. 63, no. 10, pp. 4426-4434, 2015.

[4] I. C. H. Group, C. C. Vernon, J. W. Hand, S. B. Field, D. Machin, J. B. Whaley, J. van der Zee, W. L. van Putten, G. C. van Rhoon, J. D. van Dijk, et al., "Radiotherapy with or without hyperthermia in the treatment of superficial localized breast cancer: results from five randomized controlled trials," International Journal of Radiation Oncology* Biology* Physics, vol. 35, no. 4, pp. 731-744, 1996.

[5] P. Wust, B. Hildebrandt, G. Sreenivasa, B. Rau, J. Gellermann, H. Riess, R. Felix, and P. Schlag, "Hyperthermia in combined treatment of cancer," The lancet oncology, vol. 3, no. 8, pp. 487-497, 2002.

[6] B. Enander and G. Larson, "Microwave radiometric measurements of the temperature inside a body," Electronics Letters, vol. 10, no. 15, pp. 317-317, 1974.

[7] S. Mouty, B. Bocquet, R. Ringot, N. Rocourt, and P. Devos, "Microwave radiometric imaging (mwi) for the characterisation of breast tumours," The European Physical Journal-Applied Physics, vol. 10, no. 1, pp. 7378, 2000.

[8] L. Dubois, J.-P. Sozanski, V. Tessier, J.-C. Camart, J.-J. Fabre, J. Pribetich, and M. Chive, "Temperature control and thermal dosimetry by microwave radiometry in hyperthermia," IEEE Transactions on Microwave Theory and Techniques, vol. 44, no. 10, pp. 1755-1761, 1996.

[9] S. Jacobsen and O. Klemetsen, "Improved detectability in medical microwave radio-thermometers as obtained by active antennas," IEEE Transactions on Biomedical Engineering, vol. 55, no. 12, pp. 27782785, 2008

[10] M. Lazebnik, M. C. Converse, J. H. Booske, and S. C. Hagness, "Ultrawideband temperature-dependent dielectric properties of animal liver tissue in the microwave frequency range," Physics in Medicine and Biology, vol. 51, no. 7, pp. 1941-1955, mar 2006. [Online] Available: https://doi.org/10.1088/0031-9155/51/7/022

[11] O. Fiser, M. Helbig, S. Ley, J. Sachs, and J. Vrba, "Feasibility study of temperature change detection in phantom using m-sequence radar," in 2016 10th European Conference on Antennas and Propagation (EuCAP), 2016, pp. 1-4.

[12] J. Vrba, D. Vrba, O. Fiser, I. Merunka, J. Vrba, and J. Cumana, "Microwave based medical imaging," in 2017 XXXIInd General Assembly and Scientific Symposium of the International Union of Radio Science (URSI GASS), 2017, pp. 1-4.

[13] Z. Popovic, P. Momenroodaki, and R. Scheeler, "Toward wearable wireless thermometers for internal body temperature measurements," IEEE Communications Magazine, vol. 52, no. 10, pp. 118-125, 2014.

[14] W. Haines, P. Momenroodaki, E. Berry, M. Fromandi, and Z. Popovic, "Wireless system for continuous monitoring of core body temperature," in 2017 IEEE MTT-S International Microwave Symposium (IMS), 2017, pp. 541-543.

[15] W. C. Numan, L. W. Hofstetter, G. Kotek, J. F. Bakker, E. W. Fiveland, G. C. Houston, G. Kudielka, D. T. Yeo, and M. M. Paulides, "Exploration of mr-guided head and neck hyperthermia by phantom testing of a modified prototype applicator for use with proton resonance frequency shift thermometry," International Journal of Hyperthermia, vol. 30, no. 3, pp. 184-191, 2014.

[16] D. Carter, J. MacFall, S. Clegg, X. Wan, D. Prescott, H. Charles, and T. Samulski, "Magnetic resonance thermometry during hyperthermia for human high-grade sarcoma.” International journal of radiation oncology, biology, physics, vol. 40 4, pp. 815-22, 1998.

[17] M. N. Akınc1, "Improving near-field orthogonality sampling method for qualitative microwave imaging," IEEE Transactions on Antennas and Propagation, vol. 66, no. 10, pp. 5475-5484, 2018.

[18] A. Janjic, M. Cayoren, I. Akduman, T. Yilmaz, E. Onemli, O. Bugdayci, and M. E. Aribal, "Safe: A novel microwave imaging system design for breast cancer screening and early detection-clinical evaluation," Diagnostics, vol. 11 , no. 3, p. 533, 2021.

[19] M. Haynes, J. Stang, and M. Moghaddam, "Real-time microwave imaging of differential temperature for thermal therapy monitoring," IEEE Transactions on Biomedical Engineering, vol. 61, no. 6, pp. 17871797, 2014

[20] P. M. Meaney, M. W. Fanning, K. D. Paulsen, D. Li, S. A. Pendergrass, Q. Fang, and K. L. Moodie, "Microwave thermal imaging: initial in vivo experience with a single heating zone," International Journal of
Hyperthermia, vol. 19, no. 6, pp. 617-641, 2003. [Online]. Available: https://doi.org/10.1080/0265673031000140822

[21] P. M. Van Den Berg and R. E. Kleinman, "A contrast source inversion method," Inverse problems, vol. 13, no. 6, p. 1607, 1997.

[22] J. Richmond, "Scattering by a dielectric cylinder of arbitrary cross section shape," IEEE Transactions on Antennas and Propagation, vol. 13 no. 3, pp. 334-341, 1965

[23] M. Lazebnik, D. Popovic, L. McCartney, C. B. Watkins, M. J. Lindstrom, J. Harter, S. Sewall, T. Ogilvie, A. Magliocco, T. M. Breslin, et al., "A large-scale study of the ultrawideband microwave dielectric properties of normal, benign and malignant breast tissues obtained from cancer surgeries," Physics in medicine \& biology, vol. 52, no. 20, p. 6093, 2007.

[24] M. Ambrosanio, P. Kosmas, and V. Pascazio, "A multithreshold iterative dbim-based algorithm for the imaging of heterogeneous breast tissues," IEEE Transactions on Biomedical Engineering, vol. 66, no. 2, pp. 509$520,2019$.

[25] S. Ley, S. Schilling, O. Fiser, J. Vrba, J. Sachs, and M. Helbig, "Ultrawideband temperature dependent dielectric spectroscopy of porcine tissue and blood in the microwave frequency range," Sensors, vol. 19, no. 7, 2019. [Online]. Available: https://www.mdpi.com/1424-8220/19/ $7 / 1707$

[26] A. Martellosio, M. Pasian, M. Bozzi, L. Perregrini, A. Mazzanti, F. Svelto, P. E. Summers, G. Renne, L. Preda, and M. Bellomi, "Dielectric properties characterization from 0.5 to $50 \mathrm{ghz}$ of breast cancer tissues," IEEE Transactions on Microwave Theory and Techniques, vol. 65, no. 3, pp. 998-1011, 2017.

[27] H. P. Kok, E. N. K. Cressman, W. Ceelen, C. L. Brace, R. Ivkov, H. Grüll, G. ter Haar, P. Wust, and J. Crezee, "Heating technology for malignant tumors: a review," International Journal of Hyperthermia, vol. 37, no. 1, pp. 711-741, 2020, pMID: 32579419. [Online]. Available: https://doi.org/10.1080/02656736.2020.1779357 